\title{
Analysis of Drawing and Wrinkling Control of Outer Plate of Car Side
}

\author{
Wan Zhiyuan $^{1, \mathrm{a}}$, Qiao Huaying ${ }^{1, \mathrm{~b}}$, Wei Hongmei ${ }^{1, \mathrm{c}}$ and Wang Xiaoying ${ }^{1, \mathrm{~d}}$ \\ ${ }^{1}$ School of Mechanical Engineering, Shandong Huayu University of Technology, Dezhou 253034, China \\ awzywanzhiyuan@163.com, bqqqatdj@163.com, cwhm-yf@163.com, d wxy28-10@163.com
}

Keywords: forming process, outer plate of car side, drawing, wrinkling.

\begin{abstract}
Deformation of the outer plate of the car side is very complicated in the forming process. It is difficult to grasp the deformation law and more quality problems. Based on the on-the-spot debugging, the mechanism of the wrinkling problem in the drawing process is analyzed by introducing the forming process of the outer side of the car. Wrinkling problem of outer plate of car side is solved by adjusting the shape and height of the draw bead, and also to solve the similar parts of the drawing wrinkling problem providing a reference.
\end{abstract}

\section{Introduction}

Outer plate of car side is one of the key components of the car body covering part, and the quality impact on the quality of the vehicle. Compared with the general stamping parts, it own the following characteristics. Assembly relationship is complex, as the vehicle's welding benchmark, the trunk or back door, left and right wheel cover, the floor around the longitudinal beam, left and right fender, roof with other major components have strict matching relationship [1,2] .Its geometric shape, size tolerance requirements are extremely high. Based on the lightweight and beautiful requirements, stamping sheet material is complex and surface quality requirements are high and manufacturing is difficult. As the side of the outer plate parts of these characteristics, in order to ensure that parts of the surface welding assembly consistency, inter changeability and the automotive industry, the body "2mm project" is carried out today, that the body "2mm project" is increased Side of the outer plate production difficulty[3,4]. As the side of the outer side of the whole car door surface wrinkling, there is nothing to cover, which not only affect the follow-up welding match, but also seriously affect the appearance of the vehicle quality and appearance. In this paper, it mainly analyse the wrinkling problem in the side production.

\section{Side of The Outer Plate Process Analysis}

Side of the outer plate stamping process is the most complex parts of the whole body parts, the process as shown in Table 1, and the drawing process is the most critical process stamping process. In this paper, the drawing process of a model side of the right outer plate (part model shown in Figure 1) is analyzed as an example.

Table 1 Processes

\begin{tabular}{|lrcccc|}
\hline OP05 & OP10 & OP20 & OP30 & OP40 & OP50 \\
\hline Blanking & Drawing & Trimming & Shaping & Punching and Trimming & Punching and Flanging \\
\hline
\end{tabular}

Side of the outer plate of the material is DC56D + ZF40/40, and its thickness is $0.8 \mathrm{~mm}$, which is a drawing with no gap atomic steel. The yield strength is $120-180 \mathrm{MPa}$ and the tensile strength is $260-350 \mathrm{MPa}$ and the broken elongation is not less than $37 \%$ and the part dimensions is about $2400 \mathrm{~mm} \times 1300 \mathrm{~mm} \times 230 \mathrm{~mm}$. 


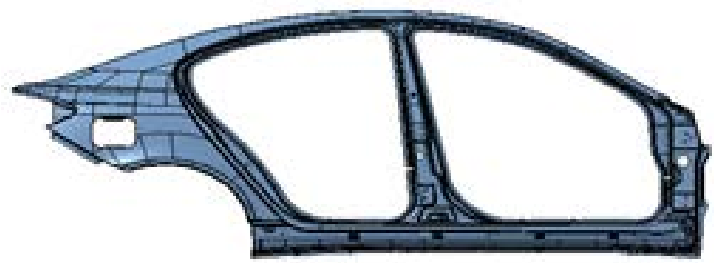

Figure 1 Part model

As shown in Figure 1, the part is very large in size and it has a very complicated structure. Therefore, the flow of the material in the process of stretching is multi-directional and difficult to control. In order to prevent all kinds of defects, the technological supplement should be added to the drawing process.

As one of the main equipment of the drawing process, the drawing die has a direct impact on the surface quality of the parts, the dimensional accuracy, the production efficiency and the economic benefits. The general drawing die structure is basically the same, which is the single-action die, and the mold size is very large, and the structure is extremely complex, which for the drawing process on-site debugging brings many problems.

\section{Analysis on the Mechanism of Drawing and Wrinkling}

Due to the complexity of the drawing process of the side plate, the main quality problems such as wrinkling, cracking and knitting are easy in the production process. Among them, wrinkling is one of the most important quality defects in the lateral drawing of the outer side plate, which is a very difficult problem in making the stamping die and determining the process parameters.

For example, the situation of sheet metal blank of the straight wall cylindrical parts is analyzed at a certain time as shown in Figure 2.

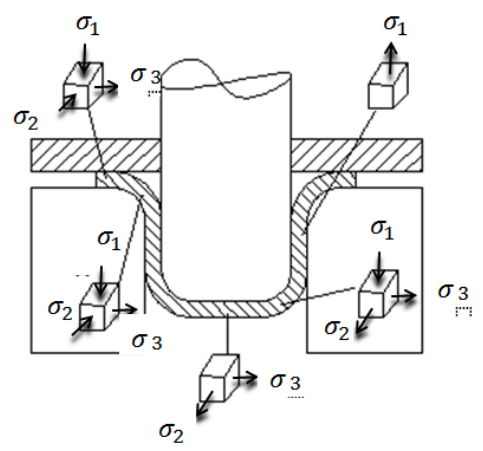

Figure 2 Rough force situation

According to the different parts of the cylindrical parts of the nature of the blank is divided into five parts [5].

(1) Plane flange part - main deformation area

It is the main deformation zone of the extensional deformation, and the fan-shaped lattice in the drawing deformation becomes a rectangular lattice region. Mainly bear radial tensile stress, tangential compressive stress and compressive stress in the direction of thickness is the two-pressure-pull three-way stress state.

(2) Die fillet part - transition zone

It is the transition between the flange and the cylinder wall part,and the material deformation is complex, the stress state and the flange part of the same, but the strain state is different, where the material thickness is thin.

(3) Cylinder wall part - force transmission area

It is formed by the deformation of the material of the flange part, and the rough thickness of the blank is 0 , and the area is in a planar state.

(4) Punches rounded parts - transition zone

The part is the transition area of the cylinder wall and the bottom of the cylinder, where the 
degree of deformation is small, and the degree of cold hardening is low, and where the material becomes thin and the cross section of the transmission force becomes smaller.So it is easy to form the "dangerous section".

(5) Bottom of the cylinder - small deformation area

The material is under the punch and is pulled into the die at the beginning of the drawing and remains in a planar shape. It subject only to radial and tangential tensile stresses. As shown in Figure 2, due to the deformation of the flange area by three-way stress, the main deformation of the sheet metal in the drawing concentrated in the drawing of the blank flange .The blank sheet by the radial tensile stress, tangential compressive stress and the thickness direction of the compressive stress three-way effect, in which the tangential compressive stress is the main cause of instability wrinkling. When the tangential compressive stress exceeds the limit of the sheet, the emergence of instability is wrinkled. From the point of view of the material mechanics, the tangential compressive stress is caused by the more serious strain of wrinkling. At the same time, the flange blanks themselves have the ability to resist the wrinkling resistance and the larger the flange width and the thinner the material and the smaller the modulus and hardening index and the less the resistance to instability.

In the final analysis, there are two reasons for wrinkling: one is that the radial blanket force is too small, because the side of the blank sheet is very large, and the local position is not pressed when the formation of tangential compressive stress is caused by wrinkling; Second, due to blank sheet metal drawing deformation, the stress is not uniform. The sheet flow speed is too large to cause instability wrinkling.

\section{Side of the outer plate drawing wrinkling problem solving}

According to the scene debugging, side of the outer door of the lower part of the door wrinkling problems shown in Figure 3. As shown in Figure 3, the press flow at the creeping position is still large. The tensile tendon does not play a role in controlling the flow of the sheet. Mold structure is shown in Figure 4.As shown in Figure 4, in the drawing die structure design, after drawing, the boundary of the part is on the outside of the drawing bar, but in the actual production, the part boundary line is inside, and the effect of the material is reduced. According to this situation, the extension of the ribs can be modified. One is that the draw bar is modified by the circular tendon to the square bar as shown in Figure 5. The second is that the circular tendon increases by $2 \mathrm{~mm}$ height as shown in Figure 6.

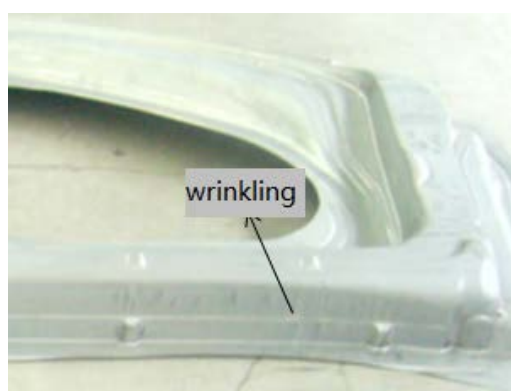

Figure 3 Part wrinkling

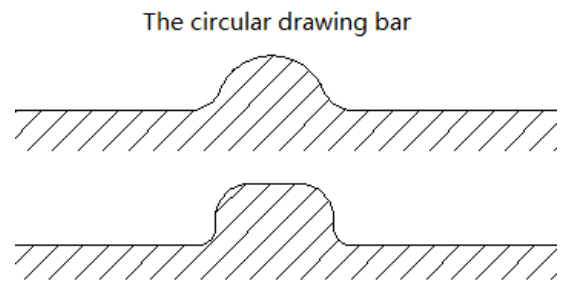

The square extension bar

Figure 5 Shape adjustment of drawing bar

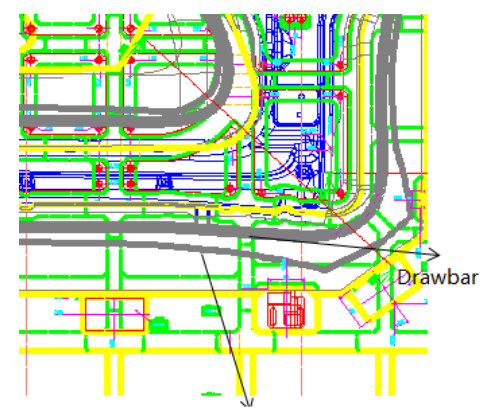

Figure 4 Mold structure

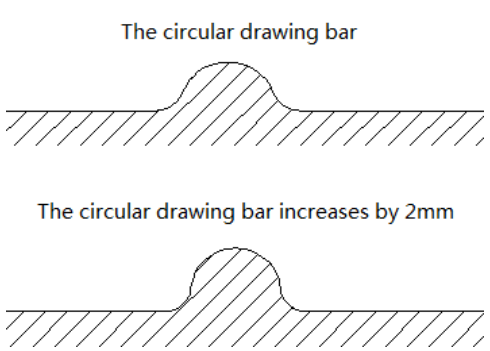

Figure 6 Height adjustment of drawing bar 
As shown in Figure 5 and Figure 6, the drawing bar is changed from circular to square or rounded heights by $2 \mathrm{~mm}$. When the sheet is deformed in the drawing, the resistance of the flow increases, and the flow rate of the sheet is effectively controlled. The wrinkling of the parts has been improved effectively. After adjusting the drawing bar, the qualified parts are finally suppressed as shown in Figure 7.

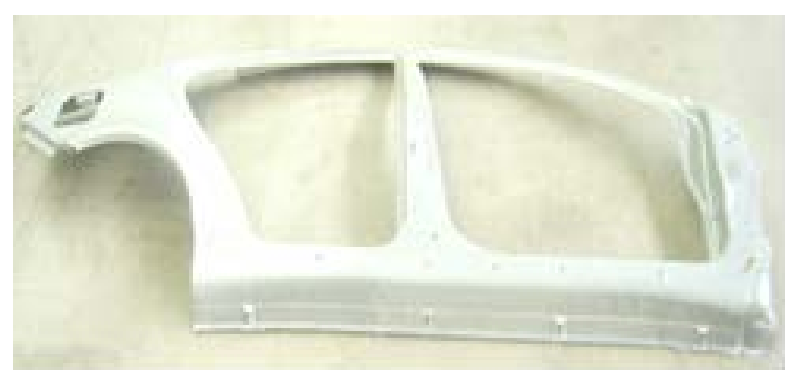

Figure 7 Qualified parts

\section{Conclusion}

Through the on-the-spot drawing and debugging, the drawing process of the outer side of the car is analyzed, and the mechanism of the wrinkling problem in the drawing process is obtained. By adjusting the drawing bar in the mold structure, the wrinkling problem in the debugging process is solved. A certain technological breakthrough has been made to the crinkle control, and finally the stability of the die state and the quality of the production is realized.

\section{References}

[1] Hao, J.W., Qin, H. I., and Wang, C. L. (2010) Inspection and treatment of cracking problem of double row integral side deep drawing. Mold Industry , 36, 36-40.

[2] Wang, Y. (2012) Research and rectification of shrinkage at the side of column. Metal processing cold processing, 7, 81-82.

[3] Yu, Y. (2010) Sheet stamping performance prediction and drawing method simulation by DYNAFORM. Jilin University.

[4] Zhang, X. F.(2009). Simulation analysis of stamping of body panel].. Wuhan University of Technology. 06, 21-23

[5] Gao, J. Z. (2001) Plastic forming process and mold design, Mechanical Industry Press. 